\title{
Effect of explicit dimensional instruction on speech category learning
}

\author{
Bharath Chandrasekaran ${ }^{1,2} \cdot$ Han-Gyol $\mathrm{Yi}^{1} \cdot$ Kirsten E. Smayda $^{2} \cdot$ W. Todd Maddox ${ }^{2}$
}

Published online: 5 November 2015

(C) The Psychonomic Society, Inc. 2015

\begin{abstract}
Learning nonnative speech categories is often considered a challenging task in adulthood. This difficulty is driven by cross-language differences in weighting critical auditory dimensions that differentiate speech categories. For example, previous studies have shown that differentiating Mandarin tonal categories requires attending to dimensions related to pitch height and direction. Relative to native speakers of Mandarin, the pitch direction dimension is underweighted by native English speakers. In the current study, we examined the effect of explicit instructions (dimension instruction) on native English speakers' Mandarin tone category learning within the framework of a dual-learning systems (DLS) model. This model predicts that successful speech category learning is initially mediated by an explicit, reflective learning system that frequently utilizes unidimensional rules, with an eventual switch to a more implicit, reflexive learning system that utilizes multidimensional rules. Participants were explicitly instructed to focus and/or ignore the pitch height dimension, the pitch direction dimension, or were given no explicit prime. Our results show that instruction instructing participants to focus on pitch direction, and instruction diverting attention away from pitch height, resulted in enhanced tone categorization. Computational modeling of participant responses suggested that instruction related to pitch direction
\end{abstract}

Bharath Chandrasekaran

bchandra@utexas.edu

1 Department of Communication Sciences and Disorders, The University of Texas at Austin, 2504A Whitis Ave., Austin, TX 78712, USA

2 Department of Psychology, The University of Texas at Austin, 2504A Whitis Ave., Austin, TX 78712, USA led to faster and more frequent use of multidimensional reflexive strategies and enhanced perceptual selectivity along the previously underweighted pitch direction dimension.

Keywords Category learning · Instruction · Computational modeling $\cdot$ Dual-learning systems

Speech categorization involves the mapping of continuous, highly variable, multidimensional acoustic cues to discrete category representations (Holt \& Lotto, 2010). Several nonnative speech categories are challenging to learn in adulthood (Iverson et al., 2003). Feature-based models attribute this difficulty to cross-language differences in the relative weighting of acoustic dimensions underlying the nonnative speech category. Despite the difficulty, there is overwhelming evidence that adults can acquire nonnative speech categories with auditory training (Bradlow, Akahane-Yamada, Pisoni, \& Tohkura, 1999a; Bradlow, Pisoni, Akahane-Yamada, \& Tohkura, 1997; Francis, Ciocca, Ma \& Fenn 2008; Francis, Kaganovich \& Driscoll-Huber 2008; Francis \& Nusbaum, 2002; Francis, Nusbaum, \& Fenn, 2007; Hattori \& Iverson, 2009; Ingvalson, Barr, \& Wong, 2013; Ingvalson, Holt, \& McClelland, 2012; Iverson, Hazan, \& Bannister, 2005a; Kondaurova \& Francis, 2010; Lively, Logan, \& Pisoni, 1993a; Lively, Pisoni, Yamada, Tohkura, \& Yamada, 1994; Logan, Lively, \& Pisoni, 1991; Wang, Jongman, \& Sereno, 2003; Wang, Spence, Jongman, \& Sereno, 1999; Wong, Perrachione, \& Parrish, 2007). In the current study we examine the impact of dimensional instruction on the acquisition of a nonnative phonetic contrast (tone). Previous studies have shown that native speakers of American English have considerable difficulty in perceiving and producing this phonetic contrast (Wang et al., 1999, 2003).

Recent work has largely focused on mechanisms underlying speech learning in adults. Mechanistically, 
attention-to-dimension (A2D) models, which derive from the generalized context model (GCM), posit that speech learning in adulthood involves attentional restructuring of the dimensions that underlie the perceptual space used in categorization (Kondaurova and Francis 2010; Francis, Ciocca et al., 2008; Francis, Kaganovich et al., 2008; Francis \& Nusbaum 2002). Learning, per A2D, involves focusing attention onto previously unattended dimensions, and/or withdrawing attention from irrelevant dimension (Kondaurova \& Francis, 2010). Attention (or withdrawal of attention) stretches (or shrinks) the perceptual space along that dimension. This allows for greater discriminability of speech sounds that vary substantially on the attended dimension. A well-studied example of a challenging speech sound category distinction is lexical tones. In tone languages like Mandarin, pitch pattern differences within a syllable can change the meaning of the word (e.g., /ma/ with a high-rising tone means "mother," while /ma/ with a high-falling tone means "to scold"). Multidimensional scaling studies show that similar acoustic dimensions underlie tone perception across languages, irrespective of the language's tonal inventory (Chandrasekaran, Gandour, \& Krishnan, 2007; Francis, Ciocca et al., 2008; Francis, Kaganovich et al., 2008; Gandour \& Harshman, 1978). However, the relative weighting of dimensions is language-dependent. For example, previous studies have found that when disambiguating tones, pitch height (average F0) and pitch direction (average slope) are two dominant dimensions identified to be used by both tone language and non-native (English) speakers (Chandrasekaran, et al., 2007; Francis, Ciocca et al., 2008; Francis, Kaganovich et al., 2008; Gandour \& Harshman, 1978). The pitch height dimension is weighted similarly by both groups; however, pitch direction is weighted more by tone language speakers, relative to English speakers, likely driven by the increased relevance of this acoustic dimension in disambiguating lexical tones in contour tone languages (Chandrasekaran, et al., 2007; Francis, Ciocca et al., 2008; Francis, Kaganovich et al., 2008; Gandour \& Harshman, 1978). Although difficult, with training, nonnative listeners can learn to increase their relative attention to pitch direction (Chandrasekaran, Sampath, \& Wong, 2010). Laboratory training paradigms largely utilize trial-bytrial feedback and/or high variability (multiple speakers) training to teach L2 speech categories (Bradlow, AkahaneYamada, Pisoni, \& Tohkura, 1999b; Lim \& Holt, 2011; Lively, Logan, \& Pisoni, 1993b; Tricomi, Delgado, McCandliss, McClelland, \& Fiez, 2006; Zhang et al., 2009; but see Iverson, Hazan, \& Bannister, 2005b; Jamieson \& Morosan, 1986). Feedback is thought to enhance learning by reducing errors, and multiple-speaker training results in learners refocusing their attention to cues that are relevant for distinguishing speech categories and/or reducing attention to irrelevant cues (Bradlow \& Bent, 2008).

The goal of this paper is two-fold. First, we examine the extent to which explicit instructions to attend to acoustic dimensions underlying lexical tone perception can impact Mandarin tone categorization in English speakers with no prior exposure to Mandarin. The role of instruction on nonnative speech learning is unclear. On one hand, several studies have shown significant learning of new auditory and speech categories with no explicit instructions provided to learners (Lim \& Holt, 2011; Vlahou, Protopapas, \& Seitz, 2012). For example, significant learning (and generalization) has been demonstrated in a videogame-based auditory training paradigm, where learning occurs incidentally by mapping between new speech categories, visual information, and motor responses (Lim \& Holt, 2011). Given these studies, we could predict that instructions will not modulate learning performance. On the other hand, more explicit training methods, such as perceptual fading, have been previously employed to train adult learners on new phonetic contrast. In this method, critical dimension(s) are exaggerated so that listeners learn to focus attention on the new dimension(s) (Jamieson \& Morosan, 1986; McCandliss, Fiez, Protopapas, Conway, \& McClelland, 2002; McClelland, Fiez, \& McCandliss, 2002). In a study examining nonspeech category learning, passive exposure to greater distributional variability along an overweighted dimension enhanced cue weighting (Holt \& Lotto, 2006). Furthermore, a phonetic training study compared various methods of modifying cue weighting, which ranged from explicit attention to critical dimensions, or increasing variability on irrelevant dimensions, found similar learning across training methods (Iverson et al., 2005a). To anticipate, we find that explicit instructions to attend to the pitch direction dimension (the dimension that is underweighted by English speakers relative to Mandarin speakers) enhances learning relative to a no-instruction control condition and two conditions that instruct listeners to focus on the pitch height dimension. A second goal of this paper was to examine the mechanisms underlying the effect of instruction. We employ computational models that allow the examination of perceptual and decisional strategies used by participants. Our models explore the processing locus of the effect of instruction and ask whether the explicit dimensional instruction affect decisional processes, perceptual processes, or both.

Our computational modeling approach derives from duallearning systems theory (DLS; Ashby, Alfonso-Reese, Turken, \& Waldron, 1998; Chandrasekaran, Koslov \& Maddox, 2014; Chandrasekaran, Yi \& Maddox, 2014; Maddox \& Chandrasekaran 2014). DLS assumes that speech 
category learning involves a competition between a reflective system that is rule-based and relies on executive function processes in the prefrontal cortex, and a reflexive system that is procedural and relies upon dopamine-mediated reward signals in the striatum (Chandrasekaran, Koslov et al., 2014; Chandrasekaran, Yi et al., 2014; Maddox \& Chandrasekaran 2014; Yi, Maddox, Mumford, \& Chandrasekaran, 2014). ${ }^{1}$ The DLS approach derives from Ashby and Townsend's (1986) general recognition theory, which is a multidimensional extension of signal detection theory (Green \& Swets, 1967). Signal detection theory postulates that behavior is determined from decisional but also perceptual processing. Signal detection theory assumes that repeated presentations of the same physical stimulus yield unique perceptual effects. Thus, over trials each physical stimulus is represented by a distribution of perceptual effects. Although the family of distribution is not specified, it is common to assume a normally distributed set of perceptual effects. Normal distributions are characterized by the mean and the variance. The mean denotes the average perceptual effect and the variance denotes the error or noise in the perceptual process.

Thus, the DLS approach dissociates perceptual from decisional processes and includes parameters that separately estimate aspects of perceptual processing from aspects of decisional processing (Maddox \& Ashby, 1996, 1998; Maddox, Ashby, \& Waldron, 2002). This allows us to determine whether explicit instruction to focus on pitch direction affects decision processes but also might lead to more accurate perceptual processing in the form of smaller perceptual noise estimates. A number of studies in the literature suggest that perceptual noise is reduced decisional forms of selectivity are operative (Goldstone 1994; Maddox 2001, 2002; Maddox et al., 2002; Maddox \& Dodd, 2003b). Given that English speakers naturally weight pitch height, we do not predict any difference in perceptual noise along the pitch height dimension as a function of explicit dimension prime condition. However, it is likely that explicit dimensional instruction to pitch direction will lead to enhanced perceptual processing along that dimension and, thus, smaller estimates of perceptual noise. We predict that explicit instruction to the pitch direction dimension will speed the transition from simple unidimensional reflective, rule-based strategies (e.g. rules related to pitch height) to a more optimal reflexive strategy that weights both dimensions (equally) during decision (Chandrasekaran, Koslov et al., 2014; Chandrasekaran, Yi et al., 2014; Maddox \& Chandrasekaran 2014; Maddox, Chandrasekaran, Smayda, \& Yi, 2013; Maddox et al., 2014).

\footnotetext{
${ }^{1}$ Although we describe the systems as prefrontal and striatal, they are more accurately defined as cortico-striatal loops. The prefrontal system involves the executive cortico-striatal loop that connects the prefrontal cortex with the head of the caudate nucleus, whereas the striatal system involves the sensorimotor cortico-striatal loop that connects high-level visual areas with the body and tail of the caudate/putamen (Alexander, DeLong, \& Strick, 1986; Seger, 2008).
}

We now briefly review the DLS model and the evidence suggesting that it underlies nonnative speech category learning.

\section{The dual-learning systems theoretical framework}

Fast and accurate categorization determines how efficiently we organize the sensory world. The systems involved in category learning have been an important focus of research (Allen \& Brooks, 1991; Ashby et al., 1998; Ashby \& Maddox, 2010; Brooks, 1978; Erickson \& Kruschke, 1998; Estes, 1986, 1994; Homa, Sterling, \& Trepel, 1981; Keri, 2003; Knowlton \& Squire, 1993; Medin \& Schaffer, 1978; Nomura et al., 2007; Nomura \& Reber, 2008; Nosofsky, 1986; Nosofsky, Palmeri, \& McKinley, 1994; Rosch, 1978; Seger \& Miller, 2010; E. E. Smith \& Medin, 1981; J. D. Smith et al., 2012). Much of what we know about the learning systems underlying category learning comes from the visual domain. An extensive body of behavioral, neuropsychological and neuroimaging work (reviewed in Chandrasekaran, Koslov et al., 2014; Chandrasekaran, Yi et al., 2014) suggests that visual category learning is mediated by at least two neural systems: a reflective (rule-based) learning system that actively generates verbal rules, is pre-frontally mediated, and relies heavily on executive function and working memory and a reflexive (procedural-based) learning system that involves predecisional integration of information across dimensions, is striatally mediated, and does not rely on executive function and working memory. When the optimal classification rule is easily verbalizable, the reflective learning system dominates. This system uses a feedback-based strategy that tests and discards rules until a rule that maximizes accuracy is uncovered. When the optimal classification rule is not verbalizable and instead is based on a predecisional integration of information, the reflexive learning system dominates. This feedback-based system learns by implicitly mapping motor responses to complex stimulus patterns as a function of reinforcement.

DLS posits that the learning systems are competitive in nature, are dissociable, and show an initial bias toward reflective (verbal rule) processing that only gives way to reflexive processing when the optimal classification rule is reflexive. Nearly all of this work has been conducted in the visual domain, but recently, this theoretical framework has been extended to speech category learning. In the next section, we address the question of whether speech category learning is reflective or reflexive optimal.

\section{Speech category learning in adulthood: reflective and reflexive}

We propose that nonnative speech category learning is optimally learned by the reflexive system. This hypothesis is 
supported by at least three lines of evidence. First, speech categories typically are difficult to verbalize, have multiple dimensions, and are highly variable. Furthermore, the redundancy and variability of cues available during speech perception prevents a simple one-to-one mapping of cues to categories (Liberman, Cooper, Shankweiler, \& Studdert-Kennedy, 1967; Lisker, 1986). Second, a recent behavioral dissociation study revealed that speech category learning was enhanced under training procedures that enhance reflexive learning and was attenuated under training procedures that enhance reflective learning (Chandrasekaran, Koslov et al., 2014; Chandrasekaran, Yi et al., 2014). Chandrasekaran, Koslov et al. (2014; Chandrasekaran, Yi et al. 2014) used the dissociation logic to investigate the extent to which lexical tone category learning in native English speakers is mediated by the reflective or reflexive learning system. We manipulated the richness of feedback, timing of feedback, and the nature of the training stimuli (high variability vs. low variability). Across all three manipulations, learning was enhanced under conditions that boost reflexive learning. For example, we found that classification accuracy was lower when the feedback provided was richer or delayed, both of which lead to worse reflexive category learning in the visual domain (Maddox, Ashby, \& Bohil, 2003; Maddox \& Ing, 2005; Maddox, Love, Glass, \& Filoteo, 2008). Classification accuracy was also lower under low-variability training, which was achieved by blocking the multiple talkers used during training. Finally, a recent neuroimaging study (Yi et al., 2014) found that successful speech category learning was associated with greater use of reflexive strategies (as determined from computational modeling) and increased activation in the putamen (a substructure within the striatum). Taken together, these data provide strong support for our claim that nonnative speech category learning is dominated by the reflexive system.

Given the competitive nature of the reflexive and reflective learning systems, one could predict that explicit instruction would not be beneficial in a reflexive-optimal task. As pointed to earlier, explicit instruction may lead to the persistence of reflective strategies, consequently lowering performance on a reflexive-optimal task. However, several lines of evidence point to the use of the reflective system early in training. For example, computational modeling studies indicate that early in training, unidimensional rule-based strategies dominate (Maddox \& Chandrasekaran 2014). Successful learners eventually transition to multidimensional learning strategies. However, in some learners, this transition from simple, reflective unidimensional strategies to reflexive multidimensional strategies does not happen. Such learners perseverate ("get stuck") with simple rules that help achieve only partial learning success. For example, older adults are poorer at learning Mandarin tone categories because they perseverate with unidimensional strategies, likely driven by an age-related deficit in executive functioning (Maddox et al., 2013). Given these findings, we predict that explicit instruction would help (not hurt) speech learning by allowing a focus of attention to the unattended dimension, thereby allowing a transition to more optimal strategies that integrate multiple dimensions.

\section{The current study}

The current study focuses on Mandarin tone learning by native English speakers under different explicit instructional conditions. Two dimensions, pitch height and pitch direction, are important for discerning tone categories across languages (see Fig. 1). For example, on the pitch height-direction continuum, the four Mandarin tone categories can be differentiated as high-level, low-rising, low-dipping, and high-falling. The pitch height dimension (average pitch across the syllable) is important for distinguishing the low tone (T3) from the high tone (T1); the pitch direction dimension is important in distinguishing rising tone (T2) from falling tone (T4). Five monosyllabic Mandarin Chinese words $(b u, d i, l u, m a$, and $m i)$ that are minimally contrasted by the four tone categories were used in the experiment, and each was produced in
Fig. 1 (Left panel) Sample fundamental frequency contours of four Mandarin tones (T1: highlevel; T2: low-rising; T3: lowdipping; T4: high-falling) produced by a male native Mandarin speaker. (Right panel) (a) Scatter plot of all stimuli. (b) Scatter plot of male-talker stimuli. (c) Scatter plot of female-talker stimuli. Stimuli dimensions (pitch height and pitch direction) were normalized between 0 and 1

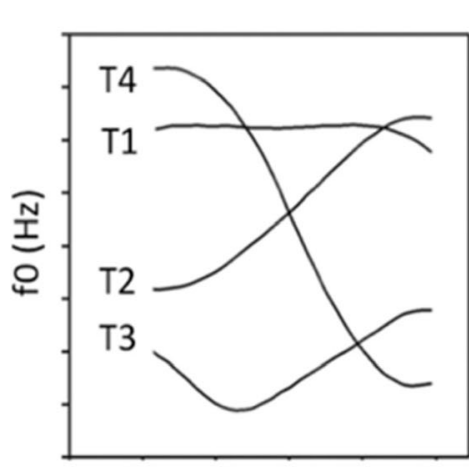

Normalized time

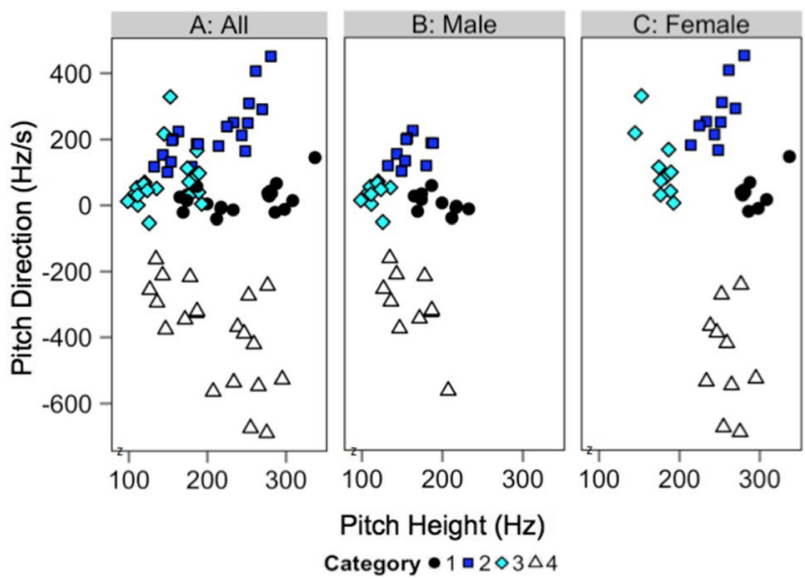


citation form with the four Mandarin tones by two male and two female native speakers of Mandarin Chinese. This yielded a total of 80 unique exemplars. A scatter plot of these 80 stimuli in the two-dimensional pitch height-pitch direction space is displayed in Fig. 1a. Scatter plots of the 40 stimuli spoken by a two male and two female speakers are displayed in Fig. $1 \mathrm{~b}$ and c, respectively. Note that no simple reflective, verbalizable rule-based strategy can be applied to accurately separate the stimuli into the four tone categories. Rather a reflexive, nonverbalizable strategy is necessary.

Across conditions (see Table 1), participants were given explicit instructions to attend to acoustic dimensions underlying tone perception. In the Control (None) condition, no explicit dimension instructions were provided. Participants were simply instructed to do their best. In the Both condition, participants were told that good task performance could be achieved by generating rules related to changes in pitch height and pitch direction. In the Height condition, participants were told that good task performance could be achieved by generating rules related to changes in pitch height. In the Direction condition, participants were told that good task performance could be achieved by creating rules related to changes in pitch direction. Additionally, in the Direction-not-Height condition, participants were told that good task performance could be achieved by creating rules related to changes in pitch direction while explicitly ignoring pitch height. As outlined earlier, pitch direction is underweighted by native English speakers but is critical to accurately disambiguate tones. We predict that participants in the Direction and
Direction-not-Height conditions will obtain superior performance relative to the Control (None) condition. In addition, as derived from the DLS theoretical approach, we also predict that the locus of this performance advantage will be due to enhanced utilization of multidimensional, reflexive decision processes that take account of both variation in pitch height and pitch direction. Specifically, DLS predicts that explicit instruction to the pitch direction dimension will speed the transition from simple unidimensional reflective, rule-based strategies (that dominate early learning) to an optimal reflexive strategy that weights both dimensions. An alternate prediction is that explicit instructions may broadly lead to a persistence of reflective strategies, resulting in lower accuracies in the speech category learning task. This prediction is based on the fact that previous studies have shown that speech learning may be reflexive optimal, and persistence of reflective strategies may not allow the transfer of control to the reflexive system. This may particularly be true for the over-weighted "height-only" condition. It is possible that explicit instruction to focus on this dimension may hurt performance more than providing no instruction at all. With respect to perceptual processes, we predict that explicit instruction to the pitch direction dimension should lead to enhanced perceptual selectivity in the form of reduced perceptual noise along that dimension. This would suggest more veridical percept of this dimension as a function of dimension prime condition. In addition, given that English speakers weight pitch height strongly even before training, we do not predict any difference
Table 1 Means and standard deviations of frequency for each tone produced by each speaker

\begin{tabular}{lllll}
\hline Speaker & Tone Category & Mean Pitch Height, Hz $(S D)$ & Mean Pitch Direction, Hz/s $(S D)$ & Sex \\
\hline 1 & 1 & $173.68(9.35)$ & $21.44(33.05)$ & Male \\
1 & 2 & $149.41(13.78)$ & $164.75(51.73)$ & Male \\
1 & 3 & $107.88(6.25)$ & $26.62(23.95)$ & Male \\
1 & 4 & $137.36(8.99)$ & $-259.82(93.87)$ & Male \\
2 & 1 & $215.60(13.83)$ & $-13.24(19.56)$ & Male \\
2 & 2 & $171.90(20.87)$ & $158.46(52.92)$ & Male \\
2 & 3 & $124.76(7.51)$ & $35.46(58.48)$ & Male \\
2 & 4 & $185.80(15.61)$ & $-354.53(146.99)$ & Male \\
3 & 1 & $278.85(2.13)$ & $35.16(6.76)$ & Female \\
3 & 2 & $242.03(14.34)$ & $243.01(59.85)$ & Female \\
3 & 3 & $170.61(24.24)$ & $149.09(155.93)$ & Female \\
3 & 4 & $266.77(24.30)$ & $-420.95(143.25)$ & Female \\
4 & 1 & $303.37(23.94)$ & $38.36(79.30)$ & Female \\
4 & 2 & $253.87(30.08)$ & $308.40(136.67)$ & Female \\
4 & 3 & $182.45(7.94)$ & $80.63(32.10)$ & Female \\
4 & 4 & $252.50(17.59)$ & $-512.26(209.20)$ & Female \\
\hline
\end{tabular}


in perceptual selectivity along the pitch height dimension as a function of dimension prime condition.

\section{Method}

\section{Participants}

One hundred fifteen individuals from The University of Texas at Austin community received course credit or were paid $\$ 8$ per hour for their participation. Informed consent was obtained from all participants, and the experiment was approved for ethics procedures using human participants. Participants who reported having learned a tone language were excluded $(n=3$ from the Both condition; $n=2$ from the Direction condition; $n=$ 1 from the Height condition; and $n=3$ from the Control condition). Therefore, a total of 106 participants were used in the final analysis, with 19 in the Control condition, 21 in the Height only condition, 20 in the Direction only condition, 21 in the Both condition, and 25 in the Direction-not-Height condition. To ensure that participants in each of our experimental conditions were homogeneous with respect to music training, given the recent results of Smayda, Chandrasekaran and Maddox (2015), we ran several one-way ANOVA's on measures of music training between conditions (Smayda et al., 2015). We found no difference between conditions for hours practiced per week, $F(4,92)=2.24, p=.07$, years played, $F(4,97)=2.20, p=.07$, number of instruments currently playing, $F(4,94)=1.94, p=.11$, and of those who played an instrument, the age they began playing, $F(4$, 43) $=0.74, p=.57$.

\section{Stimuli}

Stimuli consisted of the four Mandarin tones, Tone 1 (T1), Tone 2 (T2), Tone 3 (T3), and Tone 4 (T4) in the context of five syllables found in both Mandarin Chinese and English (bu, di, lu, ma, mi) spoken by two male and two female talkers (all originally from Beijing) for a total of 80 stimuli. All stimuli were RMS amplitude and duration normalized $(70 \mathrm{~dB}, 0.4$ s) using the software Praat (Perrachione, Lee, Ha, \& Wong, 2011; Wong, Perrachione, Gunasekera, \& Chandrasekaran, 2009). While duration and amplitude envelope are potentially useful cues to disambiguate lexical tones, behavioral studies (Howie, 1976) as well as multidimensional scaling (MDS) analyses have shown that dimensions related to pitch, especially height and direction, are used primarily to distinguish tone categories (Francis, Ciocca et al., 2008; Francis, Kaganovich et al., 2008). Therefore, we represent the stimuli used in this experiment using a space that varies on pitch height [frequency in $\mathrm{Hz}]$ and pitch direction $[(\mathrm{end} \mathrm{Hz}-$ start $\mathrm{Hz}) /$ duration (seconds)]. Five native speakers of Mandarin were asked to identify the tone categories (they were given four choices) and rate their quality and naturalness. High identification ( $>95 \%)$ was achieved across all five native speakers. Speakers rated these stimuli as highly natural. A scatter plot of the 80 stimuli in the pitch height-pitch direction space is displayed in Fig. 1a. Scatter plots of the 40 stimuli spoken by male and female speakers are displayed in Fig. $1 \mathrm{~b}$ and c, respectively. In addition, the means and standard deviations of each tone spoken by each speaker are presented in Table 1 .

\section{Procedure}

On each trial, participants were presented with a single exemplar from one of four Mandarin tone categories (T1, T2, T3, or T4) and instructed to categorize the stimulus into one of four equally likely categories. Participants were instructed that high levels of accuracy were possible but that the task would be difficult and would take practice. Participants were given corrective feedback on each trial and exposed to multiple talkers throughout the training program. No explicit instruction regarding the tone category structure was given. Participants listened to each of the 80 unique stimuli (4 tone categories $\times 5$ syllables $\times 4$ talkers) once in each block in a random order, and completed a total of six blocks of training. The task was run using the E-Prime software (Psychology Software Tools, Inc., Sharpsburg, PA), and stimuli were presented through Sennheiser HD 280 Pro headphones that were connected to the computer. Participants generated a response by pressing one of four number button keys on the left side of the computer keyboard, labeled 1, 2, 3, or 4. Following the response, feedback was provided for $1 \mathrm{~s}$ on the computer screen and consisted of the words "Correct" or "No." A 1-s ITI followed the feedback.

In each condition, we manipulated the presentation of an extra screen of instructions before participants began the task, cueing them to certain features of the stimuli. For example, the extra instructional screen for the pitch height condition read: "HINT: Prior participants have found that creating rules related to changes in the level of pitch led to good task performance." In a pilot study conducted prior to this experiment, we queried native English participants on their understanding of the terms level and direction as they relate to pitch. These terms were consistently interpreted as height ("level refers to whether pitch is high or low") and direction ("direction refers to whether pitch rises or falls"). The exact dimensional instruction for each condition can be found in Table 2. In the control condition, no extra instructional screen was presented. Critically, this is the only manipulation we made to the procedure across conditions. 
Table 2 Explicit dimension instruction presented in the extra instructional screen for each condition

\begin{tabular}{ll}
\hline Condition & Explicit Dimensional Instruction: \\
\hline Control & $\begin{array}{l}\text { (No extra screen was presented.) } \\
\text { Both }\end{array}$ \\
$\begin{array}{l}\text { "Prior participants have found that creating rules related to changes in the level of pitch and changes in pitch direction } \\
\text { led to good task performance." } \\
\text { Height }\end{array}$ & "Prior participants have found that creating rules related to changes in the level of pitch led to good task performance." \\
Direction & "Prior participants have found that creating rules related to changes in pitch direction led to good task performance." \\
Direction-not-Height & "Prior participants have found that creating rules related to changes in pitch direction led to good task performance. \\
& Prior participants have found that creating rules related to changes in level of pitch of the tone led to poor task performance."
\end{tabular}

\section{Results}

\section{Accuracy}

First, we present accuracy analyses comparing block-by-block training across the groups to determine whether the dimensional instruction had any effect on subsequent learning. Across all participants $(N=106)$, the average performance in the first learning block was $33 \%(S D=16 \%)$ and $58 \%$ $(S D=11 \%)$ in the final block. Group-by-block performance is plotted in Fig. 2.

By the final block, there was a performance advantage for the Direction $(n=20 ; M=63 \% ; S E=2.5 \%)$ and the Direction-
not-Height ( $n=25 ; M=62 \% ; S E=2 \%$ ) prime groups over the Control $(n=19 ; M=52 \% ; S E=3 \%)$ group. The Both $(n=21$; $M=55 \% ; S E=2 \%)$ and Height $(n=21 ; M=56 \% ; S E=2 \%)$ prime groups did not display a significant advantage over the Control group. To assess the validity of these observations, we employed a generalized linear mixed-effects model to analyze the results statistically, using the statistical computing package $\mathrm{R}$ (Team, 2014) in conjunction with the package lme4 (Bates et al., 2014). This analysis was designed to estimate the log odds of producing a correct response given each trial and explicit verbal prime group. The dependent variable was trial-by-trial accuracy for individual participants coded as correct or incorrect, with the reference level set as incorrect.
Fig. 2 Group-by-block performance in the speech learning task. The $x$-axis represents each successive learning block. The $y$-axis represents proportion of accurate responses per block for each group. Individual bars represent different groups. Participants in the Both and Height conditions do not perform better than the Control group, but those in the Direction and Direction-notHeight condition perform better. Error bars denote standard errors

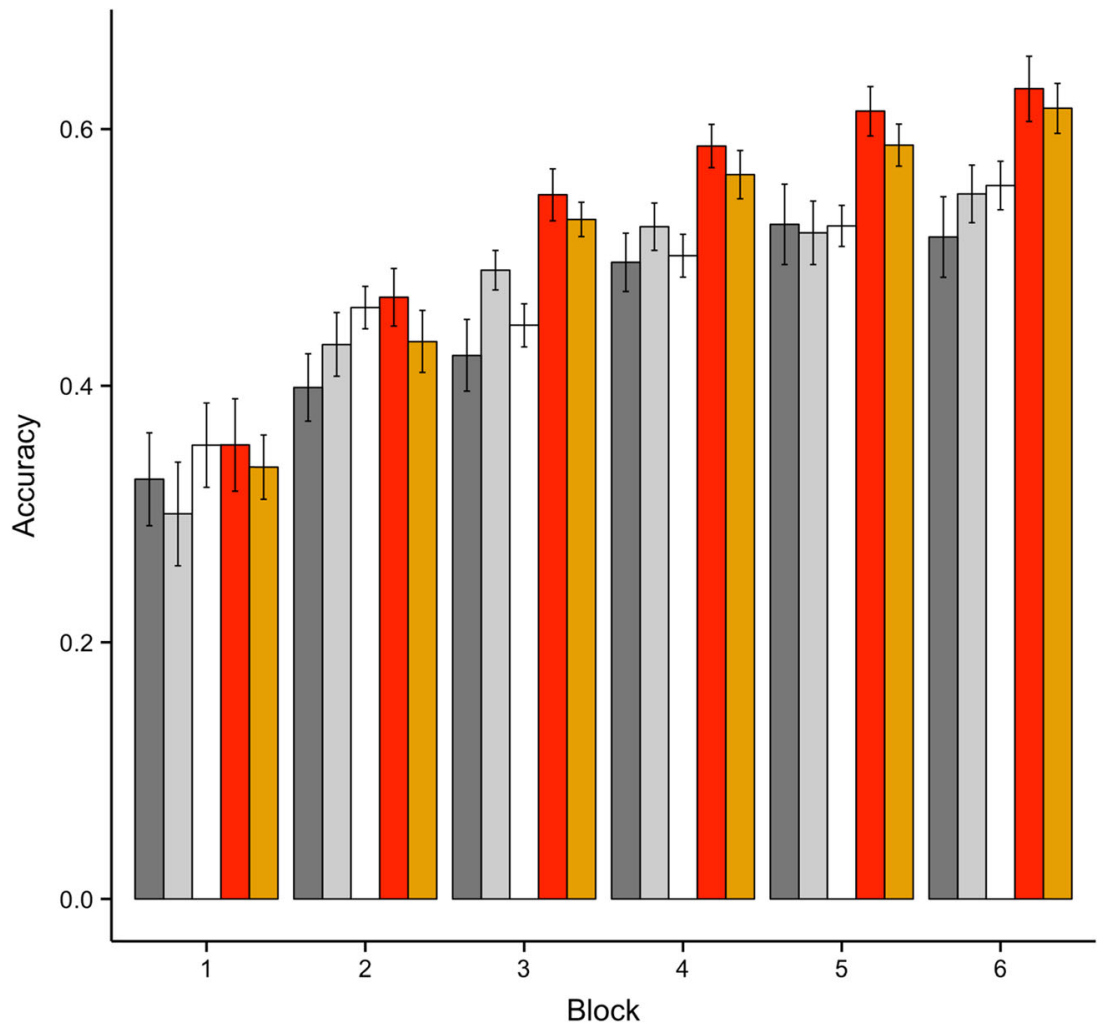

Group $\square$ Control $\square$ Both $\square$ Height $\square$ Direction $\square$ Direction-not-Height 
The fixed effects included the explicit dimensional instruction group (Control, Both, Height, Direction, Direction-notHeight, with the reference level set as Control), the trial number ( 1 to 480), and the interaction term between the two factors. The model was corrected for the random intercept for each participant. The Direction group by trial interaction was significant, $b=0.00095, S E=0.00024, z=3.95, p<.0001$, suggesting that, relative to the Control group, there was increasing odds of producing a correct response for each successive trial. In other words, the learning rate was higher for the Direction group than for the Control group. The Directionnot-Height group by trial interaction was also significant, $b=$ $0.00089, S E=0.00023, z=3.93, p<.0001$, suggesting that the learning rate for this group was higher than for the Control group. The Both group by trial interaction was not significant, $b=0.00028, S E=0.00023, \mathrm{z}=1.20, p=.23$, failing to provide evidence that the Both group had a different learning rate from that of the Control group. The Height group by trial interaction was also not significant, $b=-0.00028, S E=0.00023, z=$ $1.20, p=.23$. The trial effect was significant, $b=0.0024, S E=$ $0.00017, z=14.31, p<.0001$, attesting to the robust learning effect for each successive trial for the Control group. No group effect was found to be significant (Direction: $b=0.19, S E=$ $0.30, z=0.61, p=.54$; Direction-not-Height: $b=0.10, S E=$ $0.29, z=0.36, p=.72$; Both: $b=0.026, S E=0.30, z=0.088, p$ $=.93$; Height: $b=0.18, S E=0.30, z=0.62, p=.54$ ), suggesting that there were no initial differences in learning across groups. In summary, these results strongly suggest that the Direction and Direction-not-Height-dimension instruction led to significantly faster learning relative to the Control condition, but also that such advantage was not observed for the Both or Height dimension instructions.

\section{Computational modeling overview}

Computational modeling description The accuracy-based analyses suggest that explicit dimensional instruction to attend to pitch direction or to attend to pitch direction and ignore pitch height led to better Mandarin tone learning than instructions to attend to both dimensions, instructions to attend to pitch height, or no instructions simply to do your best. Accuracy rates provide an excellent source of information regarding how well an individual is performing in a task but tell us nothing about the perceptual and decisional processing locus of the performance advantage. To provide insights onto these important issues, we utilized computational models of the task that allow us to characterize the nature of the response strategy an individual is applying in a given task, and allow us to estimate the magnitude of trial-by-trial perceptual variability (referred to as perceptual noise). These perceptual noise estimates will allow us to determine whether verbally presented instruction affect decisional, but also perceptual processes (Goldstone, 1994; Maddox 2001, 2002; Maddox et al., 2002;
Maddox \& Dodd, 2003b). As outlined above, we predict that the Direction and Direction-not-Height instruction to speed the transition from reflective to reflexive decision strategies. Given that pitch direction is underweighted in native English speakers but is critical for Mandarin tone category learning, it is also likely that the pitch direction dimension instruction might also lead to enhanced perceptual processing in the form of less perceptual variability (noise) along the pitch direction dimension. Fortunately, the computational models provide a powerful tool for separately exploring perceptual and decisional processing and allow us to explore how perceptual and decisional processes change with experience.

Decision processing assumptions We apply a series of decision-bound models originally developed for application in the visual domain (Ashby \& Maddox, 1993; Maddox \& Ashby, 1993) and recently extended to the auditory domain by Maddox and Chandrasekaran (Maddox \& Chandrasekaran 2014; Maddox et al., 2013; Maddox et al., 2014) on a blockby-block basis at the individual participant level because of problems with interpreting fits to aggregate data (Ashby, Maddox, \& Lee, 1994; Estes, 1956; Maddox, 1999). We assume that the points in the two-dimensional (pitch height vs. pitch direction) space displayed in Fig. 1a accurately describes the average (or mean) perceptual effects for each stimulus and, based on the results from our earlier work (Maddox \& Chandrasekaran 2014), we also assume that participants operate independently on the male (Fig. 1b) and female (Fig. 1c) perceptual spaces. Our previous computational modeling studies show that native English speakers show an inherent bias toward a reflective strategy of using separate perceptual spaces by the sex of the talker, likely driven by the bias towards F0 height. The variance explained by assuming separate spaces is significantly greater than using a single perceptual space (Maddox \& Chandrasekaran 2014). We estimate parameters associated with the decision strategy being utilized by the participant. Each model assumes that decision-bounds (or category boundaries created by the participant as they learn the categories) were used to classify stimuli into each of the four Mandarin tone categories (T1, T2, T3, or T4). Note that as long as the major dimensions are known, these modeling procedures can be applied to any type of speech category structure so this offers an exciting new approach to the study of speech category learning.

Here we provide a brief description of the decision processing assumptions of each model. Details are available in numerous previous publications (Ashby \& Maddox, 1993; Maddox \& Ashby, 1993; Maddox \& Chandrasekaran 2014; Maddox et al., 2013; Maddox et al., 2014). We applied three types of models: reflexive, reflective, and random responder. The first is a computational model of the reflexive category learning system. This is instantiated with the Striatal Pattern Classifier 
(SPC; Ashby \& Waldron, 1999). The SPC is a computational model whose processing is consistent with the neurobiology of the reflexive category learning system and is thought to underlie reflexive-optimal classification performance (Ashby \& Waldron, 1999; Chandrasekaran, Koslov et al., 2014; Chandrasekaran, Yi et al., 2014; Maddox \& Chandrasekaran 2014; Maddox et al., 2013; Maddox et al., 2014; Nomura et al., 2006; Seger, 2008; Seger \& Cincotta, 2005). Responses from a hypothetical participant using the SPC are displayed in Fig. 3a. The second class is models of the reflective category learning system. The conjunctive models assume that the participant sets criteria along the pitch height and pitch direction dimensions that are then combined to determine category membership. Responses from a hypothetical participant using a conjunctive strategy are displayed in Fig. 3b. Unidimensional models assume that the participant sets criteria along the pitch height or pitch direction dimension that are then used to determine category membership. For example, the UniDimensional_Height model assumes that the participant sets three criteria along the pitch height dimension, which are used to separate the stimuli into those that are of low, medium-low, medium-high, or high pitch height. Importantly, this model ignores the pitch direction dimension. Although a large number of versions of this model are possible, we explored the eight variants of the model that made the most reasonable assumptions regarding the assignment of category labels to the four response regions. Using the convention that the first, second, third and fourth category labels are associated with low, medium-low, medium-high, and high pitch height, respectively, the eight variants were 3214 , $3412,3241,3421,2314,4312,2341$, and 4321. For example, the 3214 version of the model assumes that low pitch heights are associated with Category 3 , medium-low pitch heights are associated with Category 2, medium-high pitch heights are associated with Category 1 , and high pitch heights are associated with Category 4. Responses from a hypothetical participant using a unidimensional strategy along pitch height are displayed in Fig. 3c. The Unidimensional_Direction model assumes that the participant sets three criteria along the pitch direction dimension. The model assumes that the three criteria along the pitch direction dimension are used to separate the stimuli into those that are of low slope, medium-low slope, medium-high slope, or high pitch direction slope. Importantly, this model ignores the pitch height dimension. Although a large number of versions of this model are possible, we explored the two variants of the model that made the most reasonable assumptions regarding the assignment of category labels to the four response regions. Using the convention that the first, second, third, and fourth category labels are associated with low, medium-low, medium-high, and high pitch direction, respectively, the two variants were 4312 and 4132 . For example, the 4312 version of the model assumes that low pitch directions are associated with Category 4, medium-low pitch directions are associated with Category 3 , medium-high pitch directions are associated with Category 1, and high pitch directions are associated with Category 2. Responses from a hypothetical participant using a unidimensional strategy along pitch direction are displayed in Fig. 3d. The third model is a random responder model that assumes that the participant guesses on each trial.

Perceptual processing assumptions As outlined in the Introduction, the dual learning systems modeling framework utilized here is derived from signal detection theory. Signal detection theory assumes that behavior is a function of perceptual processing and decisional processing. The decision processing assumptions explored in this work are outlined above. Perceptually, signal detection theory assumes that repeated presentations of the same physical stimulus yield unique perceptual effects. Thus, over trials, a physical stimulus is represented by a distribution of perceptual effect. The most common assumption, and the one made here, is that the distribution is normally distributed. For simplicity and to reduce the number of free parameters in our modeling, we assume that the pitch height-pitch direction values shown in Fig. 1a-c represent the mean perceptual effects that remain unchanged throughout the task. The variability in the percepts
Fig. 3 Scatter plots of the responses along with the decision boundaries that separate response regions from a hypothetical participant using a version of the (a) Striatal Pattern Classifier, (b) Conjunctive rule-based, (c) Unidimensional_Height, and (d) Unidimensional Direction models as applied to the female talker stimuli shown in Fig. 1c

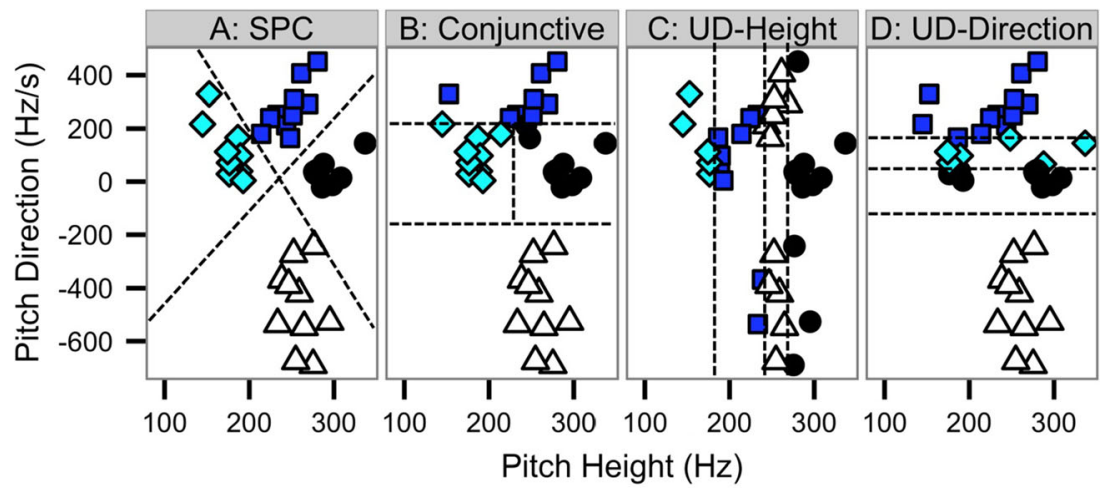

Category $\bullet 1 \square 2 \diamond 3 \Delta 4$ 
across trials is determined by estimating a perceptual variance along the pitch height dimension and a perceptual variance along the pitch direction dimension. These are also referred to as perceptual "noise" because they represent "error," or noise in the system. We assume that the perceptual variance along pitch height is identical across all 80 stimuli and that the perceptual variance along pitch direction is identical across all 80 stimuli but that these values can differ across pitch height and pitch direction (referred to as a stimulus invariant perceptual representation; Ashby \& Maddox, 1992; Maddox 2001, 2002; Maddox \& Dodd, 2003a). We also assume that the perceptual noise along the pitch height and pitch direction dimensions are uncorrelated (referred to as perceptual independence; Ashby, 1988; Ashby \& Townsend, 1986). In other words, while we estimate the perceptual variability along the pitch height dimension separately from that along the pitch direction dimension, we assume those variability estimates are constant across stimuli (stimulus invariance) and that the perceptual covariance between pitch height and pitch direction is zero (perceptual independence).

The models were fit to the Mandarin tone category learning data from each trial on a block-by-block basis by maximizing negative log-likelihood and the best fitting model was identified by comparing AIC values for each model (Akaike, 1974). AIC penalizes models with more free parameters. For each model, $i$, AIC is defined as:

$A I C_{i}=-2 \ln L_{i}+2 V_{i}$,

where $L_{i}$ is the maximum likelihood for model $i$ and $V_{i}$ is the number of free parameters in the model. Smaller AIC values indicate a better fit to the data.

\section{Computational modeling results}

The presentation of the results is divided into two parts. First, we examine decision processes by summarizing the results associated with the nature of individuals' decision strategies and how they change with experience and as a function of the explicit dimensional prime condition. Second, we examine perceptual processes. In particular we are interested in exploring how perceptual variability (noise) along the pitch direction dimension changes with experience and as a function of the explicit dimensional prime instructions.

Decisional strategies results As outlined in the Introduction section, research suggests that pitch height is a languageuniversal dimension and that native English speakers largely focus on this dimension when learning Mandarin tonal categories. With experience, however, and, we argue, under targeted instruction to focus on the pitch direction dimension, many individuals learn to spread their attention to the pitch direction dimension. Because optimal Mandarin tone learning requires the application of reflexive decision strategies that focus on both pitch height and pitch direction, we hypothesize that explicit dimensional instruction to focus on the pitch direction dimension should lead to a faster shift from reflective strategy use to reflexive strategy use. Figure 4 displays the proportion of participants in each block whose data was best fit by the reflexive, one of the three reflective or the random responder model separately by explicit dimension prime condition. A mixed effects modeling analysis was conducted with the reflexive strategy use as the dependent variable (reflexive vs. nonreflexive), and the block number, the instruction condition, and their

Fig. 4 Strategy use as estimated by computational modeling, across explicit dimensional prime groups and learning blocks. Each panel corresponds to one prime group, whereas the colors correspond to modeled response strategies. (Color figure online)

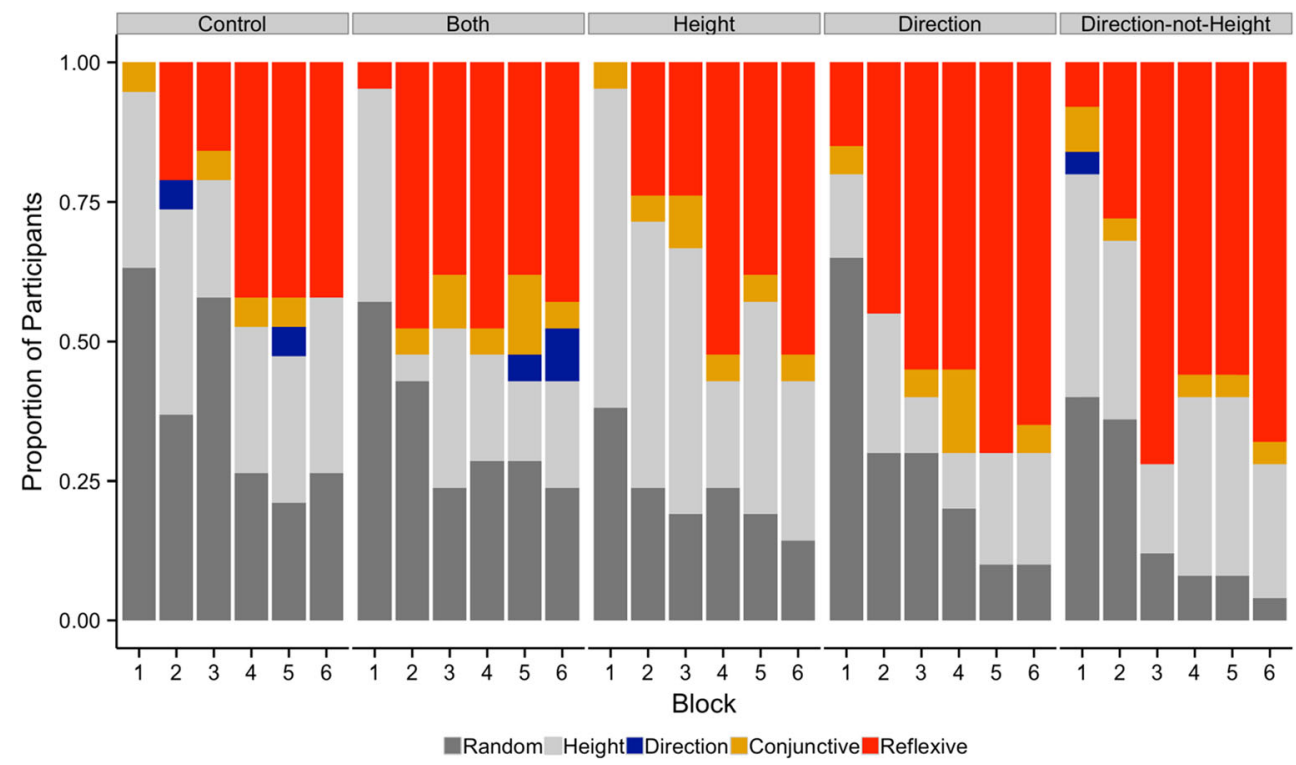


interaction terms as the fixed effects. The model was corrected for random intercepts for each participant. Relative to the Control condition, the Direction condition was associated with more reflexive strategy use, $b=$ 1.768, $S E=0.679, z=2.605, p=.009$, as well as the Direction-not-Height condition, $b=1.579, S E=0.656$, $z=2.446, p=.014$. There was no evidence that the reflexive strategy use in the Height or Both conditions were different from that in the Control condition.

As a more direct test of this hypothesis, we examined a number of aspects of the modeling results. First, we determined the first block of trials for which the SPC (a model of reflexive processing) provided the best fit of the data. If individuals given explicit dimensional instruction to focus on the pitch direction dimension show enhanced reflexive processing, then they should use reflexive strategy sooner than individuals who were not told to focus on the pitch direction dimension. Second, we determined the total number of blocks (out of six) for which the SPC provided the best account of the data. If individuals given explicit dimensional instruction to focus on the pitch direction dimension show enhanced reflexive processing, then they should use reflexive strategies (as measured by the SPC) in more blocks of trials. Data from these two measures is displayed in Fig. 5. An ANOVA examining the first block for which the SPC was utilized across pretraining instruction conditions was significant, $F(4,101)$ $=3.063, p<.05$, partial $\eta^{2}=0.108$. Post hoc analyses suggested that the SPC was used sooner in the Direction condition than in the Control (unadjusted $p=.013$; Bonferroni-corrected $p=.126$ ) and Height (unadjusted $p=.027$; Bonferronicorrected $p=.273$ ) conditions, and that the SPC was used significantly sooner in the Direction-not-Height condition than in the Control (unadjusted $p=.008$; Bonferronicorrected $p=.084$ ) and Height (unadjusted $p=.019$; Bonferroni-corrected $p=.191$ ) conditions. In addition, the first use of the SPC did not differ significantly across the Direction and Direction-not-Height conditions (unadjusted $p=.9864)$. The Both condition did not differ from the Direction (unadjusted $p=.253$ ) or the Direction-not-Height (unadjusted $p=.222$ ) condition. An examination of the total number of SPC blocks uses revealed a similar pattern. An ANOVA examining the total number of SPC blocks was significant, $F(4,101)=2.404, p=.05$, partial $\eta^{2}=0.087$. Post hoc analyses suggested that the SPC was used more often in the Direction condition than in the Control (unadjusted $p=$ .016; Bonferroni-corrected $p=.16$ ) and Height (unadjusted $p=.045$; Bonferroni-corrected $p=.45$ ) conditions, and that the SPC was used more often in the Direction-notHeight condition than in the Control (unadjusted $p=.025$; Bonferroni-adjusted $p=.25$ ) and the Height (unadjusted $p=$ .071 ; Bonferroni-adjusted $p=.71$ ) condition. In addition, the total number of SPC blocks did not differ significantly across the Direction and Direction-not-Height conditions (unadjusted $p=.754$; Bonferroni-adjusted $p=1$ ). The Both condition did not differ from the Direction (unadjusted $p=.131$ ) or the Direction-not-Height (unadjusted $p=.2$ ) condition. These findings establish that individuals given instructions to attend to pitch direction use reflexive strategies sooner and with greater regularity than individuals told to "do their best" or to "focus on pitch height."

Perceptual variance results In this section we examine the effects of explicit dimensional instruction on perceptual processing. Specifically, we ask whether explicit instruction to focus on pitch direction led to enhanced perceptual processing in the form of smaller perceptual noise estimates. A number of studies in the literature (Goldstone, 1994; Maddox 2001, 2002; Maddox et al., 2002; Maddox \& Dodd, 2003b) suggest that perceptual noise is reduced when decisional forms of selectivity are operative. Given that English speakers naturally weight pitch height, we do not predict any difference in perceptual noise along the pitch height dimension as a function of dimension prime condition. However, it is likely that explicit dimensional instruction to pitch direction should lead to
Fig. 5 Striatal pattern classifier (SPC) strategy use across groups. (Left) The first block for which the SPC provided the best account of the data in each group. Participants in the Direction and Direction-not-Height were best fit by the SPC earlier than the Control group. (Right) Total number of blocks for which SPC use was identified as the best fitting model for each group. Error bars denote standard errors
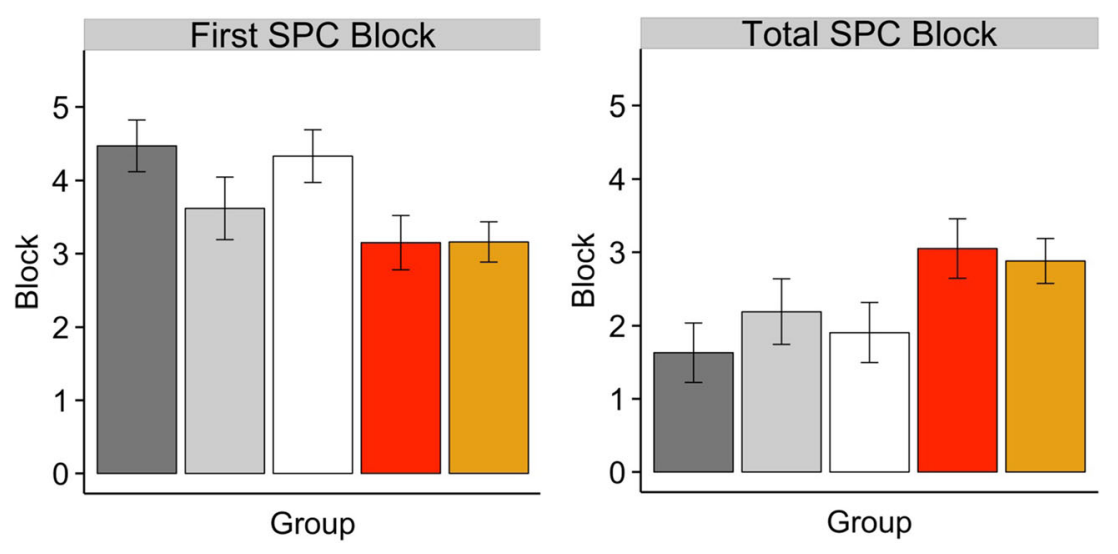

Group $\square$ Control $\square$ Both $\square$ Height $\square$ Direction $\square$ Direction-not-Height 
enhanced perceptual processing along that dimension and thus smaller estimates of perceptual noise. Because we focus on the perceptual variability estimates, we wanted to focus on the model that best accounted for the data. In the present application the most general model, and the one that provides the best account of the data, is the striatal pattern classifier (SPC). Thus, we examined the perceptual noise estimates on a block-by-block basis from the SPC for each participant.

Figure 6 displays the block-by-block average perceptual noise estimates for pitch height (Fig. 6a) and pitch direction (Fig. 6b) for each explicit dimension prime condition. A 5 dimension prime condition $\times 6$ block mixed ANOVA was conducted on the pitch height perceptual noise estimates. Mauchly's test of sphericity indicated that the assumption of sphericity had been violated, $\chi^{2}(14)=81.670, p<.001$. The main effect of block was significant, $F(5,505)=10.299$, Greenhouse-Geisser corrected $p<.001$, partial $\eta^{2}=0.093$. The main effect of condition, $F(4,101)<1.0$, and the interaction were nonsignificant, $F(20,505)=1.123$, GreenhouseGeisser corrected $p=.322$, partial $\eta^{2}=0.043$. Not surprisingly, perceptual noise estimated declined with learning but did not differ across explicit dimension prime conditions. A 5 dimension prime condition $\times 6$ block mixed ANOVA was conducted on the pitch direction perceptual noise estimates. Mauchly's test of sphericity indicated that the assumption of sphericity had been violated, $\chi^{2}(14)=71.990, p<.001$. The main effect of condition, $F(4,101)=2.823, p=.029$, partial $\eta^{2}$ $=0.101$, and block, $F(5,505)=16.242$, Greenhouse-Geisser corrected $p<.001$, partial $\eta^{2}=0.139$, were significant. The condition by block interaction, $F(20,505)=1.658$, unadjusted $p=.037$, Greenhouse-Geisser corrected $p=.055$, partial $\eta^{2}=$ 0.062 , was marginally significant. As expected, the perceptual noise estimates in the Direction and Direction-not-Height conditions were significantly smaller than in the Control condition (both Bonferroni corrected $p \mathrm{~s}<.0001$ ). In addition, the perceptual noise estimates in the Height and Both conditions did not differ significantly from the Control condition (unadjusted $p=.41$, Bonferroni corrected $p=1$; unadjusted $p=$ .011 , Bonferroni corrected $p=.109$, respectively). Perceptual noise estimates declined with experience but at different rates, depending upon the explicit dimension prime condition.

To summarize, the model-based analyses led to a number of interesting conclusions and explanations for the learning advantage associated with explicit instruction to focus on pitch direction. First, explicit instruction to focus on the (initially underweighted) pitch direction dimension led to faster and more frequent use of reflexive-optimal decision strategies relative to the conditions that included explicit instruction to focus on pitch height or no instruction. A fast transition to reflexive strategies and more consistent use of those strategies is optimal and will speed learning. Second, explicit instruction to focus on the pitch direction dimension led to enhanced perceptual processing and large reductions in perceptual noise along the pitch direction dimension relative to the conditions that included explicit instruction to focus on pitch height or no instruction. Reduced perceptual noise along the relevant pitch direction dimension is optimal and will also speed learning.

\section{Discussion}

We examined the impact of explicit dimensional instruction on the learning of Mandarin tone categories by native English speakers. We found that instruction that focused the learner toward the initially underweighted pitch direction dimension enhanced speech learning relative to instruction that focused the learning on the pitch height dimension or to a no prime control condition. Computational modeling results contextualized within the dual-learning systems model show that
Fig. 6 Block-by-block average perceptual noise estimates for (a) pitch height and pitch direction (b) for each explicit dimension prime condition

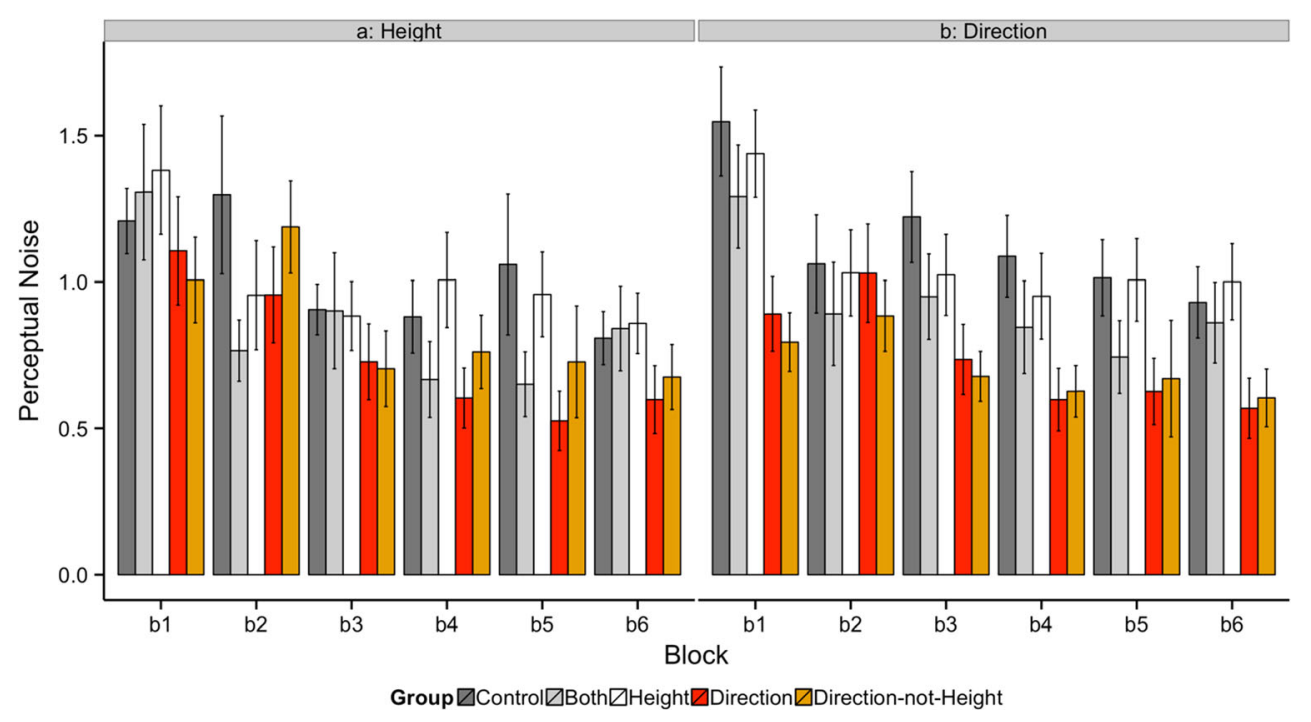


explicit dimensional instruction to focus on pitch direction affected decisional processes. From a decision processing perspective, explicit instruction to focus on pitch direction led to a faster shift from simple unidimensional reflective strategies (primarily based on the pitch height dimension) to the more optimal, multidimensional reflexive strategies, and to greater use of these reflexive strategies across learning blocks. Explicitly instructing participants to focus on already heavily weighted dimensions (in the Height only and Both conditions) did not impair performance relative to Control (no instruction). This suggests a stable, inherent bias toward using pitch height as a cue that is not modulated on the basis of instruction. Improving categorization accuracy seems to critically dependent on directing instruction toward the underweighted pitch dimension. Explicit instruction impacts perceptual processes as well; explicit instruction to focus on pitch direction led to enhanced perceptual selectivity along the pitch direction dimension, in the form of reduced perceptual noise. Although a similar reduction in perceptual noise with training was evidenced along the pitch height dimension, the reduction was not contingent on condition. Two other results are noteworthy.

The effect of explicit instruction on speech perceptual learning has not been systematically in previous research. In the speech learning studies referenced in the introduction section, participants are never cued toward dimensions. Instead, participants learn to monitor and reduce error entirely based on implicit or explicit feedback. Our results suggest that cueing to relevant dimensions may aid speech perceptual learning. A previous study examining visual category learning showed that explicit, relevant instruction on dimensions enhances category learning success only when the rules can be easily verbalized (Grimm \& Maddox, 2013). Here we find that instructing participants to attend to the pitch direction dimension is largely beneficial. It can be argued that the pitch direction dimension is a crucial and relevant dimension to successful categorization since this dimension is more resistant to talker variability. For example, the Tone 2, which is phonetically described as a low-rising tone, shows pitch movement from low to high, irrespective of the pitch of the talker. In contrast, the pitch height dimension is more impacted by talker variation (pitch height is typically higher for female talkers relative to male talkers).

\section{Theoretical implications}

It is important to note that these behavioral data are consistent with DLS but also with other theoretical approaches, namely the attention-to-dimensions approach (A2D; Francis \& Nusbaum, 2002) and the reverse hierarchy theory (RHT; Ahissar \& Hochstein, 2004). In the context of lexical tones, A2D predicts stretching and shrinking of the perceptual space along the pitch direction and pitch height dimensions, respectively. Stretching a dimension enhances the difference between two nonidentical stimuli along that dimension, making them more dissimilar and thus more easily discriminated. Shrinking a dimension attenuates the difference between two nonidentical stimuli along that dimension, making them more similar and thus more difficult to discriminate. A2D predicts that explicit instruction to pitch direction should stretch the perceptual space along that dimension, leading to improved performance, as observed. Although both approaches are consistent with the observed accuracy results, we focus the theoretical analyses on the DLS approach because it provides a computational basis for understanding the psychological processes that are affected by the explicit dimension instruction. In particular, the DLS approach dissociates perceptual from decisional processes and includes parameters that separately estimate aspects of perceptual processing from aspects of decisional processing. A2D, like other exemplar-based models, does not distinguish between perceptual and decisional influences (Maddox \& Ashby, 1996, 1998; Maddox et al., 2002). This allows us to determine whether explicit instruction to focus on pitch direction affect decision processes, perceptual processes or both. Even so, A2D is an important approach that is also consistent with the results presented in this study.

As discussed earlier, some aspects of our results are also compatible to the RHT. The RHT, like DLS was originally derived from visual perceptual learning studies (Ahissar \& Hochstein, 2004) and has subsequently been applied in the auditory and speech domain (Ahissar, Nahum, Nelken, \& Hochstein, 2009). The neurobiology of RHT and DLS differ in critical ways. In RHT, learning is instantiated within cortical regions (Ahissar et al., 2009); while DLS situates learning within corticostriatal loops (Yi et al., 2014). However, two key aspects are compatible in the context of the current study. First, RHT predicts that training initially on easy conditions (relative to hard conditions) is most beneficial to participants (Ahissar \& Hochstein, 2004); this prediction resonates with our result that providing instructions to participants enhances learning relative to the more challenging condition involving no instruction. Second, RHT predicts that expert learners are capable of switching between various levels of sensory processing (Ahissar \& Hochstein, 2004; Ahissar et al., 2009). As our computational modeling results point out, more successful learners are those who can switch seamlessly from reflective to reflexive learning processes. The DLS model differs in that the learning systems are complementary, dissociable, and learning can occur in parallel within the two systems.

\section{Explicit cueing may bootstrap reflexive learning processes}

Several lines of evidence suggest that speech categories are optimally learned through implicit, procedurallearning mechanisms. For example, in a videogame training paradigm, participants are immediately rewarded if they are accurate (Lim \& Holt, 2011; Liu \& Holt, 2011; 
Wade \& Holt, 2005). Due to the time constraints of the videogame, developing explicit rules regarding speech categories is challenging. Similarly, in a previous study we demonstrated that training manipulations that targeted implicit learning processes led to enhanced learning over trials, relative to training manipulations that targeted more explicit learning (Chandrasekaran, Koslov et al., 2014; Chandrasekaran, Yi et al., 2014). Finally, difficult categorization problems, such as the learning of the subtle Hindi retroflex-dental contrast, are achieved by incidental training approaches (Vlahou et al., 2012). Given this emerging view, our finding that explicit instructions improve speech learning is intriguing.

Our results are not incompatible with these findings. We propose that dimensional instruction may help bootstrap reflexive processes. Early in training, explicit instruction may enhance focus on a previously underutilized dimension. This may help bootstrap more implicit, reflexive learning that requires both dimensions to disambiguate categories. Furthermore, the reflexive system is critically dependent on reinforcement (via positive feedback). If a prime can increase initial success in learning via reflective learning, this would result in more positive feedback and provide scaffolding for the reflexive learning system (Crossley \& Ashby, in press). It is relevant to note that learning occurs in both systems, with a shift in relative balance determining the dominance of one system over the other (Chandrasekaran, Koslov et al., 2014; Chandrasekaran, Yi et al., 2014; Maddox \& Chandrasekaran 2014; Yi et al., 2014). Adults are inherently biased toward reflective, rule-based processes during learning. Usually, such a bias results in the development of simple rules that can be quickly tested and possibly discarded for more complex rules. The computational modeling results suggest that this is true of our current dataset as well. Irrespective of dimensional instruction, there is a tendency to predominantly use unidimensional rules at the beginning of training. In the case of Mandarin tone learning, the unidimensional rule utilizes the pitch height dimension that is already heavily weighted by native English speakers. Successful learners shift away from this simple unidimensional rule to a multidimensional, reflexive strategy that we have found is optimal for successful learning.

The development of multidimensional strategies by increasing the weighting on the pitch direction dimension is important for several reasons: tones in Mandarin vary substantially on this dimension (Wang et al., 1999, 2003), so this additional cue captures more of the variance. Additionally, the pitch direction dimension is substantially more resistant to talker variability. Shifting to a reflexive strategy may also be advantageous. This strategy places less demands on executive attention and working memory. This may allow learners to allocate cognitive resources toward other processes (e.g., vocabulary in learning novel words disambiguated by tones).

\section{Future direction}

Future studies would need to examine generalizability of these results to other speech categories. Most speech categories do not have easily identifiable dimensions or dimensions that could be primed by simple, verbalizable rules. A characteristic finding in several speech-learning studies is extreme individual variability in learning success. Based on our results, we posit that such variability could result from an inability to switch from simple, unidimensional rules to multidimensional, reflexive strategies. Thus, dimensional instruction may allow more learners to move away from suboptimal rules toward more reinforcing learning behavior. Ultimately, the key to optimal learning would most likely require individualization. For example, dimensional instruction may be most useful early in training and may have less value later in training. Thus, the duration and extent of dimensional instruction may need to be individualized in order to maximize learning potential. Another open question is whether instruction can shift perceptual weights for speech categories in the native language.

\section{Conclusion}

Our results show that dimensional instruction enhances speech learning. Critically, not all instruction enhances learning; only instruction that direct attention toward an underweighted dimension results in superior learning. Computationally, explicit dimensional instruction allows the learner to focus on an underweighted dimension, thereby developing multidimensional strategies that may capture more variance in the perceptual space and resist talker variability.

Acknowledgments This work was supported by NIDA grant DA032457 to W. T. M., and NIDCD grant DC013315 to B. C. We thank the Maddox Lab RAs and especially Seth Koslov for assistance with all data collection.

\section{References}

Ahissar, M., \& Hochstein, S. (2004). The reverse hierarchy theory of visual perceptual learning. Trends in Cognitive Sciences, 8(10), 457-464.

Ahissar, M., Nahum, M., Nelken, I., \& Hochstein, S. (2009). Reverse hierarchies and sensory learning. Philosophical Transactions of the Royal Society B: Biological Sciences, 364(1515), 285-299.

Akaike, H. (1974). A new look at the statistical model identification. Transactions on Automatic Control, 19, 716-723.

Alexander, G. E., DeLong, M. R., \& Strick, P. L. (1986). Parallel organization of functionally segregated circuits linking basal ganglia and cortex. Annual Review of Neuroscience, 9, 357-381.

Allen, S. W., \& Brooks, L. R. (1991). Specializing the operation of an explicit rule. Journal of Experimental Psychology: General, 120, 3-19. 
Ashby, F. G. (1988). Estimating the parameters of multidimensional signal detection theory from simultaneous ratings on separate stimulus components. Perception \& Psychophysics, 44, 195-204.

Ashby, F. G., Alfonso-Reese, L. A., Turken, A. U., \& Waldron, E. M. (1998). A neuropsychological theory of multiple systems in category learning. Psychological Review, 105, 442-481.

Ashby, F. G., \& Maddox, W. T. (1992). Complex decision rules in categorization: Contrasting novice and experienced performance. Journal of Experimental Psychology: Human Perception and Performance, 18(1), 50.

Ashby, F. G., \& Maddox, W. T. (1993). Relations between prototype, exemplar, and decision bound models of categorization. Journal of Mathematical Psychology, 37, 372-400.

Ashby, F. G., \& Maddox, W. T. (2010). Human category learning 2.0. Annals of the New York Academy of Sciences, 1224, 147-161.

Ashby, F. G., Maddox, W. T., \& Lee, W. W. (1994). On the dangers of averaging across subjects when using multidimensional scaling or the similarity-choice model. Psychological Science, 5(3), 144-151.

Ashby, F. G., \& Townsend, J. T. (1986). Varieties of perceptual independence. Psychological Review, 95, 124-150.

Ashby, F. G., \& Waldron, E. M. (1999). On the nature of implicit categorization. Psychonomic Bulletin \& Review, 6(3), 363-378.

Bates, D., Maechler, M., Bolker, B., Walker, S., Christensen, R. H. B., Singmann, H., . . . Rcpp, L. (2014). Package 'Ime4.' Vienna: R Foundation for Statistical Computing.

Bradlow, A. R., Akahane-Yamada, R., Pisoni, D. B., \& Tohkura, Y. (1999). Training Japanese listeners to identify English /r/ and /1/: Long-term retention of learning in perception and production. Perception \& Psychophysics, 61(5), 977-985.

Bradlow, A. R., \& Bent, T. (2008). Perceptual adaptation to non-native speech. Cognition, 106(2), 707-729.

Bradlow, A. R., Pisoni, D. B., Akahane-Yamada, R., \& Tohkura, Y. (1997). Training Japanese listeners to identify English /r/ and /1/: IV. Some effects of perceptual learning on speech production. The Journal of the Acoustical Society of America, 101(4), 2299-2310.

Brooks, L. (1978). Nonanalytic concept formation and memory for instances. Hillsdale, NJ: Erlbaum.

Chandrasekaran, B., Gandour, J. T., \& Krishnan, A. (2007). Neuroplasticity in the processing of pitch dimensions: A multidimensional scaling analysis of the mismatch negativity. Restorative Neurology and Neuroscience, 25(3/4), 195-210.

Chandrasekaran, B., Koslov, S. R., \& Maddox, W. T. (2014). Toward a dual-learning systems model of speech category learning. Frontiers in Psychology, 5, 825 .

Chandrasekaran, B., Sampath, P. D., \& Wong, P. C. (2010). Individual variability in cue-weighting and lexical tone learning. The Journal of the Acoustical Society of America, 128(1), 456-465.

Chandrasekaran, B., Yi, H. G., \& Maddox, W. T. (2014). Dual-learning systems during speech category learning. Psychonomic Bulletin \& Review, 21(2), 488-495.

Erickson, M. A., \& Kruschke, J. K. (1998). Rules and exemplars in category learning. Journal of Experimental Psychology: Learning, Memory, and Cognition, 127, 107-140.

Estes, W. K. (1956). The problem of inference from curves based on group data. Psychological Bulletin, 53, 134-140.

Estes, W. K. (1986). Array models for category learning. Cognitive Psychology, 18, 500-549.

Estes, W. K. (1994). Classification and cognition. New York, NY: Oxford University Press.

Francis, A. L., Ciocca, V., Ma, L., \& Fenn, K. (2008). Perceptual learning of Cantonese lexical tones by tone and non-tone language speakers. Journal of Phonetics, 36(2), 268-294.

Francis, A. L., Kaganovich, N., \& Driscoll-Huber, C. (2008). Cuespecific effects of categorization training on the relative weighting of acoustic cues to consonant voicing in English. The Journal of the Acoustical Society of America, 124(2), 1234-1251.
Francis, A. L., \& Nusbaum, H. C. (2002). Selective attention and the acquisition of new phonetic categories. Journal of Experimental Psychology: Human Perception and Performance, 28(2), 349-366.

Francis, A. L., Nusbaum, H. C., \& Fenn, K. (2007). Effects of training on the acoustic phonetic representation of synthetic speech. Journal of Speech, Language, and Hearing Research, 50(6), 1445-1465.

Gandour, J. T., \& Harshman, R. A. (1978). Crosslanguage differences in tone perception: A multidimensional scaling investigation. Language and Speech, 21(1), 1-33.

Goldstone, R. (1994). Influences of categorization on perceptual discrimination. Journal of Experimental Psychology: General, 123, 178200.

Green, D. M., \& Swets, J. A. (1967). Signal detection and psychophysics New York, NY: Wiley.

Grimm, L. R., \& Maddox, W. T. (2013). Differential impact of relevant and irrelevant dimension primes on rule-based and informationintegration category learning. Acta Psychologica, 144(3), 530-537.

Hattori, K., \& Iverson, P. (2009). English /r/-/1/ category assimilation by Japanese adults: Individual differences and the link to identification accuracy. The Journal of the Acoustical Society of America, 125(1), 469-479.

Holt, L. L., \& Lotto, A. J. (2006). Cue weighting in auditory categorization: Implications for first and second language acquisitiona. The Journal of the Acoustical Society of America, 119(5), 3059-3071.

Holt, L. L., \& Lotto, A. J. (2010). Speech perception as categorization. Attention, Perception, \& Psychophysics, 72(5), 1218-1227.

Homa, D., Sterling, S., \& Trepel, L. (1981). Limitations of exemplarbased generalization and the abstraction of categorical information. Journal of Experimental Psychology: Human Learning and Memory, 7, 418-439.

Howie, J. (1976). Acoustical studies of Mandarin vowels and tones. Cambridge, England: Cambridge University Press.

Ingvalson, E. M., Barr, A. M., \& Wong, P. C. (2013). Poorer phonetic perceivers show greater benefit in phonetic-phonological speech learning. Journal of Speech, Language, and Hearing Research, 56(3), 1045-1050.

Ingvalson, E. M., Holt, L. L., \& McClelland, J. L. (2012). Can native Japanese listeners learn to differentiate/r-1/on the basis of F3 onset frequency? Bilingualism, 15(2), 434-435.

Iverson, P., Hazan, V., \& Bannister, K. (2005). Phonetic training with acoustic cue manipulations: A comparison of methods for teaching English /r/-/1/ to Japanese adults. The Journal of the Acoustical Society of America, 118(5), 3267-3278.

Iverson, P., Kuhl, P. K., Akahane-Yamada, R., Diesch, E., Tohkura, Y. I., Kettermann, A., \& Siebert, C. (2003). A perceptual interference account of acquisition difficulties for non-native phonemes. Cognition, 87(1), B47-B57.

Jamieson, D. G., \& Morosan, D. E. (1986). Training non-native speech contrasts in adults: Acquisition of the English/ठ/// /contrast by francophones. Perception \& Psychophysics, 40(4), 205-215.

Keri, S. (2003). The cognitive neuroscience of category learning. Brain Research Reviews, 43(1), 85-109.

Knowlton, B. J., \& Squire, L. R. (1993). The learning of categories: Parallel brain systems for item memory and category level knowledge. Science, 262, 1747-1749.

Kondaurova, M. V., \& Francis, A. L. (2010). The role of selective attention in the acquisition of English tense and lax vowels by native Spanish listeners: Comparison of three training methods. Journal of Phonetics, 38(4), 569-587.

Liberman, A. M., Cooper, F. S., Shankweiler, D. P., \& Studdert-Kennedy, M. (1967). Perception of the speech code. Psychological Review, 74(6), 431

Lim, S. J., \& Holt, L. L. (2011). Learning foreign sounds in an alien world: Videogame training improves non-native speech categorization. Cognitive Science, 35(7), 1390-1405. 
Lisker, L. (1986). "Voicing" in English: A catalogue of acoustic features signaling/b/versus/p/in trochees. Language and Speech, 29(1), 311.

Liu, R., \& Holt, L. L. (2011). Neural changes associated with nonspeech auditory category learning parallel those of speech category acquisition. Journal of Cognitive Neuroscience, 23(3), 683-698.

Lively, S. E., Logan, J. S., \& Pisoni, D. B. (1993). Training Japanese listeners to identify English /r/ and /1/. II: The role of phonetic environment and talker variability in learning new perceptual categories. The Journal of the Acoustical Society of America, 94(3 Pt 1), 12421255.

Lively, S. E., Pisoni, D. B., Yamada, R. A., Tohkura, Y., \& Yamada, T. (1994). Training Japanese listeners to identify English /r/ and /1/. III: Long-term retention of new phonetic categories. The Journal of the Acoustical Society of America, 96(4), 2076-2087.

Logan, J. S., Lively, S. E., \& Pisoni, D. B. (1991). Training Japanese listeners to identify English /r/ and /1/: A first report. The Journal of the Acoustical Society of America, 89(2), 874-886.

Maddox, W. T. (1999). On the dangers of averaging across observers when comparing decision bound models and generalized context models of categorization. Perception \& Psychophysics, 61(2), 354-375.

Maddox, W. T. (2001). Separating perceptual processes from decisional processes in identification and categorization. Perception \& Psychophysics, 63(7), 1183-1200.

Maddox, W. T. (2002). Learning and attention in multidimensional identification and categorization: Separating low-level perceptual processes and high-level decisional processes. Journal of Experimental Psychology: Learning, Memory, and Cognition, 28(1), 99-115.

Maddox, W. T., \& Ashby, F. G. (1993). Comparing decision bound and exemplar models of categorization. Perception \& Psychophysics, 53, 49-70.

Maddox, W. T., \& Ashby, F. G. (1996). Perceptual separability, decisional separability, and the identification-speeded classification relationship. Journal of Experimental Psychology: Human Perception and Performance, 22(4), 795-817.

Maddox, W. T., \& Ashby, F. G. (1998). Selective attention and the formation of linear decision boundaries: Comment on McKinley and Nosofsky (1996). Journal of Experimental Psychology: Human Perception and Performance, 24(1), 301-321. discussion 322-339.

Maddox, W. T., Ashby, F. G., \& Bohil, C. J. (2003). Delayed feedback effects on rule-based and information-integration category learning. Journal of Experimental Psychology: Learning, Memory, and Cognition, 29(4), 650-662.

Maddox, W. T., Ashby, F. G., \& Waldron, E. M. (2002). Multiple attention systems in perceptual categorization. Memory \& Cognition, $30(3), 325-339$.

Maddox, W. T., \& Chandrasekaran, B. (2014). Tests of a dual-systems model of speech category learning. Bilingualism (Cambridge, England), 17(4), 709-728.

Maddox, W. T., Chandrasekaran, B., Smayda, K., \& Yi, H. G. (2013). Dual systems of speech category learning across the lifespan. Psychology and Aging, 28(4), 1042-1056.

Maddox, W. T., Chandrasekaran, B., Smayda, K., Yi, H. G., Koslov, S., \& Beevers, C. G. (2014). Elevated depressive symptoms enhance reflexive but not reflective auditory category learning. Cortex, 58, 186-198.

Maddox, W. T., \& Dodd, J. L. (2003). Separating perceptual and decisional attention processes in the identification and categorization of integral-dimension stimuli. Journal of Experimental Psychology: Learning, Memory, and Cognition, 29(3), 467-480.

Maddox, W. T., \& Ing, A. D. (2005). Delayed feedback disrupts the procedural-learning system but not the hypothesis-testing system in perceptual category learning. Journal of Experimental Psychology: Learning, Memory, \& Cognition, 31(1), 100-107.

Maddox, W. T., Love, B. C., Glass, B. D., \& Filoteo, J. V. (2008). When more is less: Feedback effects in perceptual category learning. Cognition, 108(2), 578-589.

McCandliss, B. D., Fiez, J. A., Protopapas, A., Conway, M., \& McClelland, J. L. (2002). Success and failure in teaching the [r]-[1] contrast to Japanese adults: Tests of a Hebbian model of plasticity and stabilization in spoken language perception. Cognitive, Affective, \& Behavioral Neuroscience, 2(2), 89-108.

McClelland, J. L., Fiez, J. A., \& McCandliss, B. D. (2002). Teaching the/ $\mathrm{r} /-/ 1 /$ discrimination to Japanese adults: Behavioral and neural aspects. Physiology \& Behavior, 77(4), 657-662.

Medin, D. L., \& Schaffer, M. M. (1978). Context theory of classification learning. Psychological Review, 85, 207-238.

Nomura, E. M., Maddox, W. T., Filoteo, J. V., Gitelman, D. R., Parrish, T. B., Mesulam, M. M., \& Reber, P. J. (2006, October 14-18). MTL and caudate contributions to visual category learning: Combining fMRI adn computational modeling. Paper presented at the Society for Neuroscience, Atlanta, GA.

Nomura, E. M., Maddox, W. T., Filoteo, J. V., Ing, A. D., Gitelman, D. R., Parrish, T. B., . . Reber, P. J. (2007). Neural correlates of rule-based and information-integration visual category learning. Cerebral Cortex, 17(1), 37-43.

Nomura, E. M., \& Reber, P. J. (2008). A review of medial temporal lobe and caudate contributions to visual category learning. Neuroscience and Biobehavioral Reviews, 32(2), 279-291.

Nosofsky, R. M. (1986). Attention, similarity, and the identificationcategorization relationship. Journal of Experimental Psychology: General, 115, 39-57.

Nosofsky, R. M., Palmeri, T. J., \& McKinley, S. C. (1994). A rule-plusexception model of classification learning. Psychological Review, 101, 53-79.

Perrachione, T. K., Lee, J., Ha, L. Y., \& Wong, P. C. (2011). Learning a novel phonological contrast depends on interactions between individual differences and training paradigm design. The Journal of the Acoustical Society of America, 130(1), 461-472.

Rosch, E. (1978). Principles of categorization. Hillsdale, NJ: Erlbaum.

Seger, C. A. (2008). How do the basal ganglia contribute to categorization? Their roles in generalization, response selection, and learning via feedback. Neuroscience and Biobehavioral Reviews, 32(2), 265-278.

Seger, C. A., \& Cincotta, C. M. (2005). The roles of the caudate nucleus in human classification learning. Journal of Neuroscience, 25(11), 2941-2951.

Seger, C. A., \& Miller, E. K. (2010). Category learning in the brain. Annual Review of Neuroscience, 33, 203-219.

Smayda, K., Chandrasekaran, B., \& Maddox, W. T. (2015). Enhanced cognitive and perceptual processing: A computational basis for the musician advantage in speech learning. Frontiers in Psychology. 6: 682. doi:10.3389/fpsyg.2015.00682

Smith, E. E., \& Medin, D. L. (1981). Categories and concepts. Cambridge, MA: Harvard University Press.

Smith, J. D., Berg, M. E., Cook, R. G., Murphy, M. S., Crossley, M. J., Boomer, J., . . . Grace, R. C. (2012). Implicit and explicit categorization: A tale of four species. Neuroscience and Biobehavioral Reviews, 36(10), 2355-2369.

Team, R. C. (2014). R: A language and environment for statistical computing. Vienna, Austria: R Foundation for Statistical Computing. Open access available at http://cran.r-project.org

Tricomi, E., Delgado, M. R., McCandliss, B. D., McClelland, J. L., \& Fiez, J. A. (2006). Performance feedback drives caudate activation in a phonological learning task. Journal of Cognitive Neuroscience, 18(6), 1029-1043.

Vlahou, E. L., Protopapas, A., \& Seitz, A. R. (2012). Implicit training of nonnative speech stimuli. Journal of Experimental Psychology: General, 141(2), 363. 
Wade, T., \& Holt, L. L. (2005). Incidental categorization of spectrally complex non-invariant auditory stimuli in a computer game task. The Journal of the Acoustical Society of America, 118(4), 2618-2633.

Wang, Y., Jongman, A., \& Sereno, J. A. (2003). Acoustic and perceptual evaluation of Mandarin tone productions before and after perceptual training. The Journal of the Acoustical Society of America, 113(2), 1033-1043.

Wang, Y., Spence, M. M., Jongman, A., \& Sereno, J. A. (1999). Training American listeners to perceive Mandarin tones. The Journal of the Acoustical Society of America, 106(6), 3649-3658.

Wong, P. C., Perrachione, T. K., Gunasekera, G., \& Chandrasekaran, B. (2009). Communication disorders in speakers of tone languages:
Etiological bases and clinical considerations. Seminars in Speech and Language, 30(3), 162-173.

Wong, P. C., Perrachione, T. K., \& Parrish, T. B. (2007). Neural characteristics of successful and less successful speech and word learning in adults. Human Brain Mapping, 28(10), 995-1006.

Yi, H. G., Maddox, W. T., Mumford, J. A., \& Chandrasekaran, B. (2014). The role of corticostriatal systems in speech category learning. Cereb Cortex. doi:10.1093/cercor/bhu236

Zhang, Y., Kuhl, P. K., Imada, T., Iverson, P., Pruitt, J., Stevens, E. B., . . . Nemoto, I. (2009). Neural signatures of phonetic learning in adulthood: A magnetoencephalography study. NeuroImage, 46(1), 226240 . 